Service social

\title{
Parents d'adolescents : un défi parfois difficile
}

\section{Micheline Beaudet et Céline Bégin}

Volume 35, numéro 3, 1986

Les jeunes et le travail social

URI : https://id.erudit.org/iderudit/706317ar

DOI : https://doi.org/10.7202/706317ar

Aller au sommaire du numéro

\section{Éditeur(s)}

École de service social de l'Université Laval

\section{ISSN}

1708-1734 (numérique)

Découvrir la revue

\section{Citer cet article}

Beaudet, M. \& Bégin, C. (1986). Parents d’adolescents : un défi parfois difficile. Service social, 35(3), 339-351. https://doi.org/10.7202/706317ar

\section{Résumé de l'article}

Après nous avoir fait partager leur réflexion sur l'adolescence, les relations parents-enfants et les relations entre pairs, les auteures décrivent le mouvement Toughlove, né aux États-Unis il y a une quinzaine d'années. Elles présentent ensuite une expérience québécoise et en font l'évaluation. d'utilisation que vous pouvez consulter en ligne.

https://apropos.erudit.org/fr/usagers/politique-dutilisation/ 
BEAUDET, Micheline, étudiante à la maîtrise à l'École de service social de l'Université Laval.

BÉGIN, Céline, travailleuse sociale au Centre d'accueil Louis-Hébert de Québec.

\section{Parents d'adolescents : un défi parfois difficile}

\section{Micheline Beaudet Céline Bégin}

"Les pères s'habituent à laisser leurs enfants faire ce qu'ils veulent. Et ils ont peur de dire quoi que ce soit à leurs rejetons. Quant aux fils, ils veulent le rôle de leurs pères: ni crainte, ni souci de ce que disent leurs parents. Leur seul désir est l'indépendance de l'adulte. Les maîtres eux-mêmes, dans les circonstances actuelles, tremblent devant leurs élèves. Ils préfèrent les flatter au lieu de les mener d'une main ferme sur une voie normale, et les adolescents finissent par mépriser les adultes.

"Nous en sommes à l'âge où les plus jeunes se comparent aux aînés, les contestent par la parole et l'action. La génération des Anciens, elle, cherche à plaire à sa postérité. Elle feint d'ignorer la stupidité ou la désobéissance de la jeunesse, elle y prend même part pour ne pas donner l'impression que les "vieux" sont des trouble-fête ou des inquisiteurs.

"Ainsi l'âme et la force morale des jeunes se dégradent-elles peu à peu. Ils se rebellent jusqu'à ne plus pouvoir supporter la moindre marque de subordination. À la limite, ils méprisent les lois. Nul ne voudra admettre que quelqu'un ou quelque chose lui soit supérieur. Voilà comment débute juvénilement et joyeusement la tyrannie."

(Platon, La République, [4e s. av. J.-C.].) 
On croirait entendre des parents de 1986 ou un groupe de professeurs de polyvalente lors d'une journée pédagogique ! Ce texte, écrit il y a 23 siècles, a de quoi laisser songeur... Il n'y aurait donc pas de "solution miracle" ou de réponse définitive aux mutations culturelles ou au conflit des générations? Plus ça va, plus c'est pareil ! Mais cette constatation, si elle a le pouvoir de rendre modestes nos initiatives, n'empêche ni les efforts ni, surtout, l'espoir.

Cet article présente une expérience d'intervention auprès de parents en difficulté avec leurs jeunes. La première partie esquisse un cadre général de l'adolescence et la deuxième vise à faire connaître l'approche de groupe du mouvement Toughlove.

\section{L’adolescence}

\section{La durée de l'adolescence}

L'adolescence n'est pas un phénomène nouveau ; il y a toujours eu une phase de transition entre l'enfance et l'âge adulte. Les sociétés, d'hier et d'aujourd'hui, ont résolu et doivent résoudre, d'une façon ou d'une autre, le défi qui consiste à transformer des enfants en adultes : leur survie et leur bon fonctionnement dépendent des solutions choisies, compte tenu des objectifs de ces sociétés et des valeurs qui les supportent.

Lorsque la civilisation avait un caractère agraire, les besoins des jeunes étaient nécessairement subordonnés à la survie économique, et les occupations rudimentaires leur permettaient d'atteindre rapidement la productivité des adultes. Les milieux institutionnels qui les formaient étaient la famille et leur lieu de travail. En bref, la "socialisation" se concrétisait tôt par l'interaction continuelle avec les parents et autres adultes.

Mais la société moderne, avec ses structures occupationnelles plus complexes, a suscité des périodes de formation de plus en plus longues, de telle sorte que les jeunes sont "gardés » le plus longtemps possible à l'école et à l'extérieur du marché du travail ; c'est encore plus vrai en temps de crise économique. L'adolescence constitue donc actuellement une phase plus étendue que par le passé et plus difficile à situer dans le temps. Quand se termine l'enfance exactement? et quand commence l'âge adulte?

On considère généralement qu'il y a trois dimensions : biologique, psychologique et sociale, à l'adolescence, mais il est délicat de la délimiter. Néanmoins, le point de départ semble faire l'unanimité: 
le début de la puberté inaugure un processus nouveau dans le cycle de la vie, engage le jeune dans une étape différente de ce qu'il avait vécu jusque-là et ferme le monde de son enfance.

Si le début semble évident pour tout le monde, adolescents comme parents, il n'en est pas de même pour la fin, ce qui soulève d'innombrables difficultés, à plusieurs niveaux : sociétal, familial et individuel. En effet, si l'adolescence s'achève par l'insertion du jeune dans le monde des adultes, la reconnaissance de ce passage ne fait l'objet d'aucune manifestation publique. Peter Blos, qui peut être considéré comme un spécialiste de l'adolescence, fait remarquer qu'aucun indicateur précis n'indique la fin de cette période. Toutefois, on « interprète " généralement la conjonction d'événements sociaux et de facteurs de maturité psychologique comme l'entrée dans le monde adulte; avoir quitté l'école, accéder au marché du travail, se détacher de sa famille, s'engager dans une vie de couple, établir un milieu de vie indépendant, seraient des indicateurs sociaux "visibles " de l'évolution psychologique qui se manifeste à l'adolescence : rompre certains liens de dépendance à l'égard des parents, acquérir des capacités d'intimité sexuelle, affirmer une certaine identité du moi.

II n'y a pas si longtemps, un quart de siècle à peine, ces événements sociaux se déroulaient, dans la majorité des cas, comme des expériences concomitantes ou rapprochées. C'est du moins la "référence" de la plupart des parents d'adolescents : une trajectoire continue entre travail, mariage et nouvelle famille. Aujourd'hui, cependant, avec le phénomène du décrochage scolaire, un marché du travail synonyme d'emplois occasionnels de courte durée, la précocité de l'activité sexuelle, distincte de l'engagement, et l'éducation continue, qui oserait prétendre que ces indicateurs annoncent un changement définitif, le passage vers un état permanent?

\section{Les relations parents-adolescents}

Il n'y a pas "un" modèle d'interaction parents-adolescents: la variété de ces relations est aussi large que celle de n'importe quelle situation interpersonnelle. Certains adolescents ont des rapports harmonieux avec leurs parents, sans conflit majeur ; d'autres ne désarment pas, peu importent les enjeux, petits ou grands. Les tâches qui leur permettent de se développer exigent une implication croissante dans une vie extra-familiale et leurs besoins sont marqués d'une grande ambivalence. Ainsi, on remarque qu'ils recherchent à la fois l'autonomie et la sécurité, l'adhésion à un groupe de pairs et une certaine originalité, l'identification à des personnes significatives tout en restant eux-mêmes, 
des expériences diverses idéalement couronnées de succès. En peu de mots, ils cherchent une identité qui oscille entre l'envie d'être unique et la peur d'être différent.

À cette étape, plusieurs jeunes commencent à ressentir qu'ils sont devenus des adultes et que, désormais, ils n'ont plus besoin de supervision parentale. Ils fonctionnent comme si leur famille avait peu d'influence sur eux et leur comportement. Toutefois, même si cette période en est une d'indépendance accrue, les attitudes familiales continuent de jouer un rôle important dans leur développement.

Le problème central, pour les parents d'adolescents, pourrait se formuler comme suit : comment aider les adolescents à acquérir graduellement leur autonomie et leur indépendance tout en maintenant certaines contraintes et limites nécessaires à des comportements qui ne sont pas arrivés à maturité? Devant ce problème, on retrouve une gamme d'attitudes fort différentes:

- autocratique : l'adolescent ne peut pas exprimer ses vues et ses opinions et il ne participe à aucune des décisions qui le concernent ;

- autoritaire : l'adolescent peut contribuer à la solution d'un problème qui le concerne mais doit s'en remettre à la décision finale de ses parents et à leur jugement ;

- démocratique : l'adolescent est encouragé à participer activement aux décisions familiales même si l'approbation finale revient à ses parents ;

- égalitaire : ici, il y a peu de différences entre les parents et les enfants; les deux générations ont un droit de parole égal dans les affaires familiales;

- permissive : l'adolescent a de plus grandes responsabilités que ses parents dans les domaines qui le concernent ;

- laissez-faire: l'adolescent a le choix de tenir compte ou de rejeter les souhaits de ses parents dans ses prises de décision;

— indifférente : les parents sont complètement désintéressés du comportement de leur adolescent et de ce qu'il devient.

On voit bien, par cet éventail d'attitudes parentales, qu'il y a oscillation entre les frontières de la liberté et du contrôle. Globalement, on pourrait placer ces attitudes à deux extrêmes : d'un côté, le refus du changement, caractérisé par le maintien des règles et des modes de communication de la période antérieure et par une restriction de l'implication du jeune avec ses pairs (système trop fermé); de l'autre, l'abandon exagéré du contrôle, où, sous prétexte du respect de l'autonomie de l'adolescent, on ne retrouve plus de cadre de référence, ce qui engendre la confusion (système trop ouvert). 
Les relations entre parents et adolescents sont donc soumises à une influence réciproque où le comportement des uns exerce des pressions et contrôle celui des autres: une discipline autoritaire qui inclut la punition physique est associée à la dépendance et à la révolte chez les adolescents; une discipline trop permissive peut également conduire aux mêmes effets, aussi paradoxal que cela puisse paraître.

Les recherches révèlent qu'il y a peu de différences réelles entre les attitudes et valeurs des adolescents et celles de leurs parents. Les premiers, cependant, ont tendance à surestimer cet écart et les derniers à s'en inquiéter !

\section{Les relations avec les pairs}

Dans ses relations avec les pairs, l'adolescent expérimenterait son identité, comme si ses semblables "légitimaient " et " corroboraient " sa démarche et sa recherche avant qu'il ait à la justifier à l'extérieur de son groupe d'appartenance, à ses parents et à d'autres adultes. On remarque alors un conflit entre l'influence des parents et celle des pairs. Ce déplacement d'allégeance préoccupe énormément les parents qui observent des attitudes nouvelles chez leurs jeunes, dont la propension à approuver, du moins extérieurement, les conduites antisociales d'autres jeunes.

L'esprit de clan et l'intolérance envers ceux qui sont différents permettent à l'adolescent de clarifier temporairement qui il est, en précisant ce qu'il n'est pas. L'habillement et l'apparence physique constituent les insignes de son identification à un modèle culturel spécifique (disco, rock, punk, etc.) et au style de musique qu'il préfère. Ils adoptent souvent un dialecte particulier qui accentue la cohésion de leur groupe et les distance des adultes. Ce dialecte varie d'une génération à l'autre et n'indique pas, en soi, une incapacité à maîtriser sa langue.

En somme, l'adolescent, via son groupe social, tente de réconcilier les deux grandes forces conflictuelles de tout être humain : l'orientation vers l'individualité et le besoin d'appartenance sociale.

Les Chinois, pour écrire le mot " crise ", doivent utiliser deux signes distinctifs : l'un signifie " possibilité, opportunité " et l'autre, "danger ". Les parents qui vivent la crise d'adolescence comme une phase de déséquilibre, de contestation, ne sont pas exempts d'inquiétudes et de préoccupations mais, tout compte fait, ils s'appuient sur des acquis qui les rassurent, entre autres sur une communication « régulière » avec les jeunes. Vivre avec des adolescents les oblige à répondre presque quotidiennement à des questions sur la sexualité, leurs certitudes et leurs valeurs et les oblige à se redéfinir; cette introspection n'est pas 
une sinécure! Toutefois, certains autres parents font face à des comportements qui les alarment et leur font pressentir un danger : c'est pour eux que le mouvement d'entraide Toughlove a été créé.

\section{Le mouvement d'entraide Toughlove}

Ce mouvement, né aux États-Unis, regroupe des parents qui ont des difficultés avec leurs adolescents et leurs jeunes adultes. Ces jeunes présentent des comportements qui sont loin d'être reconnus comme typiques de cette étape de vie, et les parents se sentent démunis, effrayés, sans ressources et isolés.

Toughlove a suscité une gamme de réactions dont l'enthousiasme, l'inquiétude et la méfiance. Certains critiques ont parlé de "virage à droite ", de retour à une époque, supposément révolue en éducation, où la discipline, dans son sens le plus étroit, est remise à l'honneur. Ils ont aussi parlé de parents qui mettent leurs jeunes à la porte. Laurent Laplante, dans un article du quotidien Le Soleil, traduit ce terme par " le rude amour "; et les parents du groupe de la Maison de la famille se baptisent quelquefois, avec humour, "les parents terribles", d'après l'expression d'un jeune qui, voyant ses parents partir pour la rencontre hebdomadaire du groupe, leur a dit : "vous allez encore retrouver vos parents terribles!»

Comme Toughlove inquiète surtout ceux qui le connaissent peu ou mal, en voici la petite histoire, racontée à partir des questions les plus souvent posées.

\section{Qu'est-ce qu'un groupe de parents utilisant le modèle Toughlove?}

Toughlove est un groupe d'entraide de parents qui éprouvent des difficultés avec leurs jeunes et qui se supportent pour agir sur les comportements problématiques de ces derniers. Selon le modèle d'intervention prôné, ils doivent :

— identifier ces comportements problématiques ;

- prendre position (cette position doit être commune lorsque les parents vivent ensemble) face à l'un de ces comportements ;

- établir une limite à court terme (une semaine) et dresser un plan d'action incluant une sanction si la limite n'est pas respectée.

Pour ce faire, les parents sont aidés, à chacune de ces étapes, par les autres parents qui constituent leur groupe de support. Le support 
mutuel est l'un des éléments les plus remarquables de ce modèle d'intervention, les parents s'entraidant non seulement pendant les rencontres mais également par des contacts téléphoniques entre les rencontres. Ils peuvent aussi être appelés à faire des démarches dans le milieu : accompagner un parent à la Cour, préparer et négocier un contrat avec un jeune, etc.

\section{Quels parents peuvent en bénéficier?}

Le groupe est accessible à tous les parents dont les jeunes (12 ans à 25 ans) présentent des problèmes de comportement majeurs, c'està-dire :

- rentrent à la maison ivres ou drogués,

- empruntent de l'argent sans le rendre,

- volent,

- fuguent,

- refusent systématiquement de participer aux tâches domestiques,

- sont violents, verbalement ou physiquement,

- ont atteint la majorité et refusent d'étudier, de travailler, de prendre leurs responsabilités.

Pour que les parents puissent travailler avec le groupe, il faut aussi que le jeune habite à la maison ou y passe au moins les fins de semaine, s'il fait l'objet d'un placement temporaire.

\section{Comment ce mouvement a-t-il commencé ?}

Le premier groupe Toughlove a été mis sur pied aux États-Unis, il y a une quinzaine d'années, par deux parents: David et Phyllis York. Intervenants familiaux d'expérience, ils avaient élevé leurs trois filles selon les bons principes du docteur Spock et selon les valeurs éducatives privilégiées dans leur milieu, c'est-à-dire qu'ils comptaient quasi uniquement sur l'amour et la compréhension pour assurer à leurs filles une croissance harmonieuse.

Comme beaucoup de parents, ils ont rencontré certaines difficultés lorsque leurs filles ont atteint l'adolescence. Ils ont alors eu recours aux formes d'aide accessibles, et se sont même engagés dans des thérapies individuelles pour identifier en quoi les conflits non résolus de leur enfance pouvaient expliquer leurs défaillances en tant que parents. Cependant, ils ont dû faire face à une situation d'urgence lorsque les policiers se sont présentés à leur domicile pour arrêter leur fille cadette, 
âgée de 17 ans, accusée d'avoir menacé un revendeur de drogue avec une arme à feu. Dépassés par la situation et fatigués de recoller les pots cassés, ils ont fait appel à des amis pour trouver une solution à ce cauchemar.

Le premier groupe Toughlove était né. Des décisions concrètes ont été prises: les parents ne paieraient pas le cautionnement et la jeune fille passerait les vacances de Noël en prison. Pour une fois, elle assumerait les conséquences de ses actes. Les York, se sachant incapables de la visiter en prison sans succomber à ses demandes, ont demandé à leurs amis de les remplacer auprès d'elle. Ces derniers l'ont visitée, lui ont apporté vêtements et réconfort et ont négocié les conditions de sa sortie, qui incluaient un séjour dans un centre de désintoxication. Évidemment, les York ont dû résister aux pressions de leurs deux autres filles, en total désaccord avec ces décisions.

Le mouvement a fait boule de neige et il existe maintenant environ 1200 groupes dans le monde, majoritairement en milieux anglophones. Dans la région de Montréal, il y a six groupes francophones.

À Québec, nous avons démarré le premier groupe en novembre 1984 dans le cadre du programme de prévention de la délinquance du Service de réadaptation sociale. Il a immédiatement obtenu le support de la Maison de la famille de Québec et fait toujours partie de sa programmation. Nous avons pu compter sur le support des professeurs en intervention de groupe de l'École de service social de l'Université Laval. Actuellement, ce groupe pilote fonctionne de façon autonome et trois autres ont été mis sur pied et bénéficient d'un encadrement fourni par des intervenantes des C.L.S.C. : de la Jacques-Cartier, de la Côte de Beaupré et Laurentien, avec la coopération de la Polyvalente de l'Ancienne-Lorette. Deux autres groupes sont actuellement à l'étape du recrutement : l'un au C.L.S.C. de Beauport et l'autre dans un contexte de pratique privée, à Sainte-Foy.

\section{Quels sont la philosophie de base et les buts de ces groupes?}

\section{Philosophie}

Les initiateurs de l'approche Toughlove en résument la philosophie par les dix principes de base suivants :

- les problèmes familiaux ont des racines culturelles;

- les parents sont aussi des êtres humains; 
- il y a des limites aux capacités matérielles et émotives des parents ;

- les parents et les enfants ne sont pas égaux;

- le comportement des parents influence celui des enfants; le comportement des enfants influence celui des parents ;

- blâmer entretient un sentiment d'impuissance ;

- prendre position provoque une crise ;

- une crise bien contrôlée amène un changement positif ;

- les familles ont besoin de donner du support à leur communauté et d'en recevoir de celle-ci ;

- la base de la vie familiale est la coopération et non pas l'unité de ses membres.

\section{Buts}

Les interventions du groupe visent à amener les parents à changer leurs propres comportements, les seuls sur lesquels ils ont du pouvoir puisqu'ils ont perdu le contrôle de la vie familiale. Le groupe les aide à modifier leurs rôles et à s'entendre sur des actions communes. Ce faisant, ils provoquent une crise chez leur jeune : ce dernier est forcé de constater que ses comportements antérieurs ne sont plus efficaces. II doit donc changer lui-même ou assumer les conséquences de ses actions inacceptables.

Ce travail se fait en vue d'une réconciliation éventuelle. On ne veut pas briser la relation entre les parents et le jeune en difficulté mais aider la famille à trouver de nouveaux modes d'interaction satisfaisants pour tous. Cependant, on ne recherche pas une réconciliation à tout prix et il arrive que le jeune choisisse de briser la relation; dès lors, les autres membres de la famille n'ont plus à subir ses comportements destructeurs. On peut cependant espérer que le temps et les expériences vécues par le jeune, à l'extérieur, l'amènent à renouer des liens avec sa famille sur des bases différentes.

\section{Comment se déroulent les rencontres?}

Les rencontres hebdomadaires durent deux heures et demie. Les parents qui vivent en couple doivent y participer ensemble. Elles sont très structurées et comportent quatre parties :

- un retour sur les objectifs choisis la semaine précédente (20 minutes); 
- une période d'information (40 minutes), réservée d'abord aux échanges concernant le fonctionnement du groupe (par exemple : l'attribution des rôles). Elle est aussi consacrée à l'acquisition de connaissances nouvelles sur des thèmes choisis par les participants : drogue, besoins des adolescents, participation aux tâches, etc. Le groupe fait alors fréquemment appel à des intervenants qui, tout en apportant un contenu répondant aux besoins des parents, prennent connaissance de cette méthode. Certains thèmes sont travaillés sans apport de l'extérieur, par le biais de lectures ou de discussions;

- le travail sur les objectifs ( 40 minutes). Chaque parent se donne, pour la semaine qui débute, une tâche précise en rapport avec le comportement problématique à modifier chez son jeune. Il est encouragé à expérimenter de nouvelles façons d'agir avec lui pour le surprendre et susciter des réponses différentes. Les couples sont invités à inverser les rôles, le parent le plus engagé devant céder l'action à son conjoint. Les techniques de travail préconisées par l'approche sont : la confrontation avec support, le brainstorming, la préparation de contrats avec le jeune et la planification du support d'autres parents jusqu'à la rencontre suivante, surtout par le biais de contacts téléphoniques;

- l'accueil des nouveaux membres, le travail sur les situations difficiles, les jeux de rôles et une brève évaluation de la rencontre (20 minutes).

Les rencontres sont animées par les parents, qui se partagent aussi les rôles de coordonnateur, de trésorier, de préposé à l'accueil et de "teneur de temps", la durée des interventions étant limitée pour permettre à chacun de travailler. Les rôles les plus exigeants sont assumés par les parents les plus anciens et les plus expérimentés. Souvent, ils ont résolu leurs propres problèmes et restent dans le groupe pour aider les autres, comme le demande Toughlove. Ils y consolident alors leurs acquis et font des apprentissages personnels importants.

\section{Les interventions du groupe sont-elles efficaces?}

D'après les évaluations faites auprès de parents qui ont persévéré au moins trois mois dans leur travail avec le groupe, des changements importants se manifestent dans les comportements des jeunes.

De plus, enrichis de connaissances nouvelles et de nouveaux apprentissages, les parents semblent rassurés et plus adéquats dans 
leurs relations avec leurs jeunes. Il est évident que, lorsque les interactions deviennent mieux adaptées à l'étape de vie de la famille (celle où les parents doivent accepter que leurs jeunes prennent des responsabilités de plus en plus grandes et en assument les conséquences, pas nécessairement heureuses), les relations s'améliorent, non seulement entre les parents et le jeune concerné mais entre tous les membres de la famille. Autre fait important, ils ne se sentent plus isolés et sans ressources pour jouer leurs rôles difficiles de parents, rôles que la société leur reconnaît et leur confie sans leur apporter les appuis nécessaires pour les remplir avec une certaine aisance. On peut même constater que les valeurs véhiculées actuellement (hédonisme, remise en question de l'autorité) et les problèmes nouveaux (drogues, sexualité précoce) ont souvent une influence négative sur le vécu familial, en grande partie parce que les parents ne peuvent se référer à leur propre vécu.

Comme Toughlove préconise la coopération avec la communauté : écoles, policiers, intervenants sociaux, les parents apprennent à aller vers des ressources nouvelles. Il reste cependant que ce modèle d'intervention s'est montré inefficace avec certains parents, en particulier :

- ceux qui présentent des difficultés de couple si importantes qu'ils n'arrivent pas à s'entendre pour faire face aux difficultés de leurs jeunes;

- ceux qui ne suivent pas la démarche du groupe de façon régulière ;

- ceux qui ne mettent pas en pratique les décisions prises avec le groupe.

Le groupe a aussi rencontré des difficultés dans son travail avec les parents qui, ayant vécu seuls avec leurs enfants pendant une longue période après la mort du conjoint ou une séparation, ont réinvesti dans une relation de couple sans que le nouveau "conjoint " habite d'une façon régulière avec la famille. Souvent, ce dernier ne veut pas intervenir dans l'éducation des jeunes, décision qui, en pratique est bien difficile à maintenir.

\section{Cette intervention présente-t-elle des risques?}

Cette question est habituellement posée en rapport avec des situations dramatiques : le jeune est dépressif, a menacé ou tenté de se suicider; il y a de la violence ou une menace sérieuse de violence ; il est question de demander à un jeune ayant atteint la majorité de choisir entre l'adoption de comportements acceptables ou le départ de la maison. 
Toughlove demande, dans ces situations à risques élevés, d'user de la plus grande prudence. Le groupe n'intervient pas dans la problématique du suicide et exige que les parents communiquent avec une ressource appropriée (par exemple : le Centre de prévention du suicide) avant de travailler à d'autres niveaux. Quant à la violence physique, elle est fermement proscrite, à l'exception des situations où il faut restreindre les mouvements du jeune ou l'immobiliser lorsqu'il a perdu le contrôle de lui-même et menace de tout casser. Les parents qui craignent d'être frappés par leurs enfants sont incités à éviter les situations explosives et à prévoir du secours : voisins, autres parents, policiers ; car les parents battus, ça existe aussi.

L'expérience vécue à Québec incite aussi à la vigilance concernant l'acceptation de parents qui ne correspondent pas à la clientèle-cible décrite par les York : les parents ayant éduqué leurs jeunes avec une grande permissivité et peu d'encadrement. Toughlove a attiré quelques parents qui maintenaient un style d'autorité parentale adapté à des enfants plus jeunes mais inadéquat pour des adolescents. Attirés par la philosophie du mouvement qui, à première vue, soutient leur position, ils ont été confrontés par les participants et amenés à laisser plus d'autonomie à leurs enfants. Cependant, si ces parents étaient majoritaires dans un groupe, les prises de position seraient inappropriées et même inacceptables. Les groupes doivent être sensibilisés à cette éventualité.

Cette forme d'intervention n'est pas à l'abri de suites malheureuses. Elle présente des risques relatifs; cependant, dans les situations familiales en cause, ne pas intervenir présente aussi des risques importants. II est tout de même surprenant de constater que le "défaut d'agir " cause, la plupart du temps, moins d'inquiétude qu'une action planifiée.

Et, sans vouloir faire le procès des autres formes d'intervention de pratique professionnelle, peut-on dire qu'elles ne présentent pas de risques, ou moins de risques?

\section{Conclusion}

On nous a demandé d'écrire cet article pour présenter une nouvelle forme d'intervention auprès des familles qui rencontrent des difficultés avec leurs adolescents et leurs jeunes adultes. Nous avons cru bon de rappeler certaines dimensions caractéristiques de l'adolescence et du contexte social dans lequel elle se déroule.

Nous croyons que les parents sont les premiers intervenants auprès de leurs enfants et que toute action entreprise auprès des jeunes n'a des 
chances de changement durable qu'en autant que les parents ne sont pas oubliés.

En ce qui nous concerne, après avoir côtoyé de près tant de familles "souffrantes" et démunies, nous avons démystifié les maléfices des mots "contrôle" et "pouvoir " des parents; de toute façon, lorsqu'ils n'exercent pas leur pouvoir légitime, ce sont les jeunes qui s'empressent de se l'approprier, à leur plus grand détriment.

\section{Références bibliographiques}

Btos, Peter, The Adolescent Passage : Developmental Issues, New York, International Universities Press, 1979, 521p.

Hopkins, J. Roy, Adolescence, the Transitional Years, New York, Academic Press, 1983, 494p.

LAPLANTE, Laurent, "La formule du rude amour", Le Soleil, 12 novembre 1984, p. B-4.

YORK, Phyllis et David YORK, Thoughlove: a Self-Help Manual for Parents Troubled by Teenage Behavior, (édition revisée), Doylestown (Penn.), Toughlove Inc., 1984, 97p.

YORK, Phyllis, David York et T. WACHTEL, Toughlove, Garden City (N.Y.), Bantam Books, 1982, 210p. 